\title{
Acral papulovesicular eruption to smallpox vaccination
}

\section{Lesley Hawley $^{1 *}$, Adam Perry ${ }^{1}$, John Childs ${ }^{1}$ and Aubrey Winn ${ }^{2}$}

${ }^{1}$ Naval Medical Center San Diego, USA

${ }^{2}$ Walter Reed National Military Medical Center, USA

\section{Introduction}

This is a case of a unique reaction to the ACAM2000 ${ }^{\circ}$ Small Pox vaccination previously reported in one case series. We find the morphology and locations of this reaction to be very interesting and appeared clinically consistent with a cutaneous hypersensitivity versus vasculitic reaction on patient presentation.

\section{Report of a case}

A 27 year-old man with an unremarkable medical history received pre-deployment anthrax, MMR, Typhoid, Hepatitis B, and ACAM $2000^{\circ}$ Small Pox vaccinations on February 08, 2013. He reported an expected course with a vaccination site blister on his left upper arm with associated lymphadenopathy and malaise on post vaccination day (PVD) 3 which resolved by PVD 7. This was followed by the appearance of erythematous tender papules on his dorsal hands and fingers. By PVD14 multiple erythematous blanching papules measuring 2-5 mm in diameter were present on the dorsal hands, lateral fingers, extensor elbows, and knees (Figure 1). He had no atypical targetoid lesions and no oral or ocular involvement was present. The vaccination site on his left upper arm was well healed with no residual crust or drainage. There was no preceding viral respiratory infection, myalgia, pharyngitis, gastrointestinal upset or difficulty breathing and he had no reported contact with ill individuals following immunizations. Laboratory analysis showed a normal ESR, CRP, CBC, Chem 18, and ANA. Biopsy specimens were obtained from papular lesions on his right elbow and right dorsal hand.

\section{Diagnosis}

Acral Papulovesicular Eruption Secondary to Smallpox Vaccination

\section{Microscopic findings}

Biopsy showed mild spongiosis with focal vacuolar degeneration along the dermal-epidermal junction. In the dermis there was papillary dermal edema and a moderately dense superficial to mid-dermal perivascular and perieccrine lymphocytic infiltrate (Figure 2). There was one focal area of epidermal necrosis and no leukocytoclastic vasculitis. PCR was not performed on the biopsy specimen.

\section{Discussion}

Small pox is a life threatening disease caused by an orthopoxvirus with a mortality rate reported as high as $30 \%$ [1]. In December 2002, the United States implemented a program of smallpox vaccinations for approximately 500,000 military personnel which came as part of a national program of preparedness against biological attack [2]. Because of the virus' long incubation period, the vaccine can be given up to 3 days after exposure and still prevent disease, however, preventative vaccination was determined to be the best way to avoid mass casualty

and loss of mission completion. Dryvax, a calf lymph derived vaccine which contains the NYCBOH vaccinia strain, was initially used in 2002 and yielded similar adverse event statistics as those reported in the 1968 national surveillance data, including incidence of an exanthematous reaction pattern of $.054 \%$ [3]. In March 2008, ACAM $2000^{\circ}$ replaced Dryvax $^{\oplus}$ as the vaccine used and stockpiled in the United States to protect against the threat of smallpox. ACAM 2000 contains the same vaccinia strain as Dryvax but is grown in African green monkey cells and the vehicle used was thought to be less reactogenic in comparison
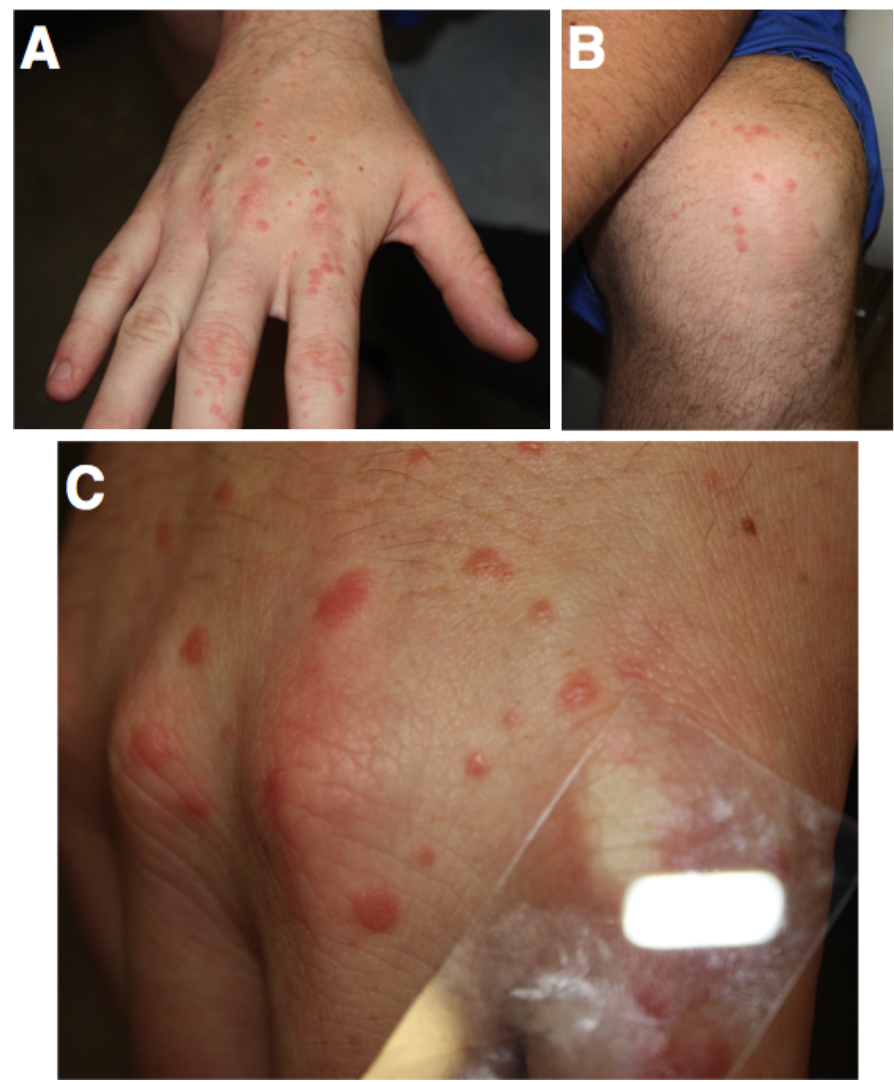

Figure 1. Multiple erythematous blanching papules measuring $2-5 \mathrm{~mm}$ in diameter were present on the A) dorsal hands, lateral fingers, and B) knees. C) Note diascopy demonstrating blanching nature of papules.

Correspondence to: Lesley Hawley, Department of Dermatology, Naval Medical Center San Diego, 34800 Bob Wilson Drive, San Diego, CA 92134, USA, Tel: 619-315-9656; E-mail: hawley.lesley@gmail.com

Received: December 23, 2014; Accepted: January 15, 2015; Published: January 18,2015 


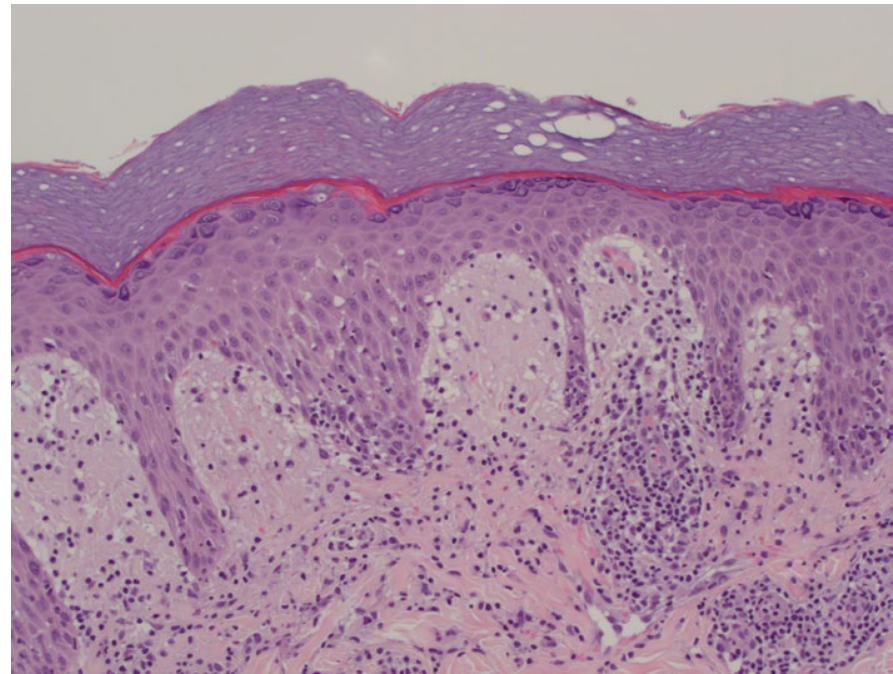

Figure 2. Mild spongiosis with focal vacuolar degeneration along dermal-epiderma junction. Noted papillary dermal edema and a moderately dense superficial to mid-dermal perivascular and perieccrine lymphocytic infiltrate.

to first and second generation vaccines [3,4]. Initial data suggests very similar rates, with approximately $11 \%$ of ACAM $2000^{\circ}$ smallpox vaccination recipients reporting cutaneous reactions in locations away from the vaccination site, compared to $10 \%$ with those vaccinated using Dryvax [5].

The induction of protective immunogenicity has been shown in animal trials to result from both humoral and cellular immune responses. Adverse reactions range in severity and typically involve skin; including urticaria, autoinoculation, erythema multiforme and nonspecific dermatidites. Potentially more serious adverse effects include but are not limited to eczema vaccinatum, generalized vaccinia, Stevens Johnson Syndrome, myocarditis and encephalitis [6]. This particular case appears to represent a unique response pattern that has been previously referred to as a benign, acral papulovesicular eruption. These specific symptoms thought to be associated with ACAM $2000^{\circ}$ administration have been reported in one previous case series [5]. Our current case, as well as the aforementioned case series, show striking clinical and histologic similarity but are confounded by the fact that multiple vaccinations were received along with ACAM $2000^{\circ}$. Though a causal relationship between smallpox vaccination and the appearance of acral vesiculopustular eruptions cannot be definitively proven, the coadministered vaccines have not been reported to cause the cutaneous reaction pattern described above. Our patient did not require treatment and symptoms resolved by PVD 20.

\section{References}

1. Bessinger GT, Smith SB, Olivere JW, James BL (2007) Benign Hypersensitivity Reactions to Smallpox Vaccine. International J Derm 46: 460-465. [Crossref]

2. Baciu A, Anason A (2005) The Smallpox Vaccination Program: Public Health in an Age of Terrorism. Washington D.C.: The National Academies Press.

3. Cono J, Casey CG, Bell DM (2003) Smallpox Vaccination and Adverse Reactions Recommendations and Reports. Center for Disease Control. 52 (RRo4).

4. Lane JM, Goldstein J (2003) Evaluation of $21^{\text {st }}$ Century Risks of Smallpox Vaccination and Policy Options. Ann Intern Med 138: 488-493. [Crossref]

5. Beachkofsky TM, Carrizales SC, Bidinger JJ, Hrncir DE, Whittemore DE, et al. (2010) Adverse Events Following Smallpox Vaccination With ACAM2000 in a Military Population. Arch Dermatol 146: 656-661. [Crossref]

6. Tack DM, Karem KL, Montgomery JR, Collins L, Bryant-Genevier MG, et al. (2013) Unintentional transfer of vaccinia virus associated with smallpox vaccines: ACAM2000(®) compared with Dryvax(®). Hum Vacc Immunother 9: 1489-1496. [Crossref]

Copyright: (C)2015 Hawley L. This is an open-access article distributed under the terms of the Creative Commons Attribution License, which permits unrestricted use, distribution, and reproduction in any medium, provided the original author and source are credited. 